\title{
Tightening force and torque of nonlocking screws in a reverse shoulder prosthesis
}

\author{
A. Terrier ${ }^{\text {a,* }}$, S.H. Kochbeck ${ }^{\text {a }}$, F. Merlini ${ }^{\text {a }}$, M. Gortchacow ${ }^{\text {a }}$, D.P. Pioletti ${ }^{\text {a }}$, A. Farron ${ }^{\text {b }}$ \\ a Laboratory of Biomechanical Orthopedics, Ecole Polytechnique Fédérale de Lausanne, Switzerland \\ b Department of Musculoskeletal Medicine, University Hospital Center and University of Lausanne, Switzerland
}

\section{A R T I C L E I N F O}

\section{Article history:}

Received 20 August 2009

Accepted 23 March 2010

Available online $\mathrm{xxxx}$

\section{Keywords:}

Shoulder

Arthroplasty

Reversed prosthesis

Screw

Cadaveric study

\begin{abstract}
A B S T R A C T
Background: Reversed shoulder arthroplasty is an accepted treatment for glenohumeral arthritis associated to rotator cuff deficiency. For most reversed shoulder prostheses, the baseplate of the glenoid component is uncemented and its primary stability is provided by a central peg and peripheral screws. Because of the importance of the primary stability for a good osteo-integration of the baseplate, the optimal fixation of the screws is crucial. In particular, the amplitude of the tightening force of the nonlocking screws is clearly associated to this stability. Since this force is unknown, it is currently not accounted for in experimental or numerical analyses. Thus, the primary goal of this work is to measure this tightening force experimentally. In addition, the tightening torque was also measured, to estimate an optimal surgical value.

Methods: An experimental setup with an instrumented baseplate was developed to measure simultaneously the tightening force, tightening torque and screwing angle, of the nonlocking screws of the Aquealis reversed prosthesis. In addition, the amount of bone volume around each screw was measured with a micro-CT. Measurements were performed on 6 human cadaveric scapulae.

Findings: A statistically correlated relationship $(p<0.05, R=0.83)$ was obtained between the maximal tightening force and the bone volume. The relationship between the tightening torque and the bone volume was not statistically significant.

Interpretation: The experimental relationship presented in this paper can be used in numerical analyses to improve the baseplate fixation in the glenoid bone.
\end{abstract}

(c) 2010 Elsevier Ltd. All rights reserved.

\section{Introduction}

The reversed shoulder arthroplasty is a recently accepted treatment for rotator cuff arthropathy. The medial displacement of the glenohumeral rotation center increases the moment arm of the remaining muscles, and the conforming articular surface provides the joint stability that is normally achieved by the rotator cuff muscles. The loosening of the glenoid component was a severe drawback of the original design (Boileau et al., 2005). This problem has been significantly improved in recent designs (Boileau et al., 2006), despite some reported loosening cases (Sirveaux et al., 2004; Wierks et al., 2009). However, because of the constrained nature of the prosthesis and the limited bone volume available, the optimal fixation of the glenoid component remains an open question (Chebli et al., 2008).

A stable initial fixation is crucial for the long term success rate of cementless implants (Soballe et al., 1992; Kwong et al., 1994; Kienapfel et al., 1999). This is also true for the glenoid metallic baseplate of reversed prostheses. Baseplate stability is indeed usually achieved by a central peg and peripheral screws (Bohsali et al., 2006; Guery et al., 2006). The optimization of the screw position has been recently investigated using

\footnotetext{
* Corresponding author. Laboratory of Biomechanical Orthopedics, Station 15, Ecole Polytechnique Fédérale de Lausanne, 1015 Lausanne, Switzerland.

E-mail address: alexander.terrier@epfl.ch (A. Terrier).
}

micro-CT reconstructions of cadaveric scapulas (Humphrey et al., 2008). In this paper, the authors underlined the lack of information regarding the tightening force that reflects the actual load seen at the interface between bone and the baseplate. To bypass this problem, they used assumptions obtained from spine surgery studies, but actually did not account for the tightening force. They concluded that optimal screws fixation could be achieved in 3 major columns of the scapula: the base of the coracoid, the spine of the scapula and the pilar. In another study, the glenosphere lateralization was analyzed together with the screw size on the different prostheses inserted in blocks of artificial bone (Harman et al., 2005). The study concluded that the baseplate stability decreased with lateralization, but could be improved with larger screws. Another study used artificial scapulae of uniform polyurethane foam to estimate the initial stability of the baseplate (Codsi and Iannotti, 2008). Screw force was not measured, but it was concluded that the posterior screw should be directed towards the spine and the anterior screw should be directed inferior to the central peg. It confirmed that maximizing bone support of compression screws improves baseplate stability. Another experiment with two artificial bone qualities and 5 different screw configurations showed that 4 screws were better than two and more eccentric screw positioning improved stability (Roche et al., 2008). Finally, numerical methods were used to reproduce and confirm experimental measurement stating that baseplate stability was improved by increasing the length, diameter and inclination of the fixation screws (Harman et al., 2005; Hopkins et al., 2008). 
The above biomechanical analyses on the baseplate fixation are quite few and none of them considered the tightening (compressive) screw force. This force is however critical for the initial stability of the baseplate. An estimate of this force, as close as possible to real clinical situation would thus be very useful in the stability analysis of the baseplate. In a real human glenoid bone, this force is obviously related to the tightening torque applied by the surgeon. This surgical tightening torque is obtained by the experience of the surgeon, who can feel the approaching limit of the maximum torque before bone failure. It is also quite obvious that this maximal torque is related to the amount of bone surrounding and supporting the screw.

Therefore, the goal of this study was to obtain a relationship between the tightening force, the tightening torque, and the bone volume surrounding the screw. For this purpose, an experimental setup was developed to measure simultaneously the tightening screw force, the tightening torque, the screwing angle and the bone volume around the nonlocking compression screw with cadaveric scapulae. The results of this paper will be used later to optimize the number and location of the compression screws that stabilize the glenoid baseplate.

\section{Methods}

\subsection{Cadaveric samples}

Six unpaired cadaveric scapulae (Anatomy Department, University Hospital of Lausanne) with unknown gender and age were used. There was no sign of degenerative disease (arthritis or osteoporosis) in any scapula. We dissected the scapulas from cadavers that were perfused with a solution (0.91 formaldehyde, 0.51 phenol, 11 glycerin, 10.61 water). The scapulae were then stored at $-20{ }^{\circ} \mathrm{C}$ and thawed at $+20{ }^{\circ} \mathrm{C}$ $24 \mathrm{~h}$ before the measurements.

\subsection{Surgical procedure}

The Aequalis reversed shoulder prosthesis was used for this study (Tornier, Inc. Medina, MN, USA). The baseplate positioning was performed as in the operation room, by an experience surgeon, using the prosthesis instrumentation. The glenoid bone was prepared as recommended by the manufacturer. After the reaming of the glenoid bone, the central hole (for the central peg) was drilled. The baseplate central peg was then press-fitted into the central hole. When the baseplate was positioned, the holes for the anterior and posterior nonlocking screws were drilled. The surgeon replicated the screws positioning and drilling technique (with the same instrumentation) as performed in patients of our orthopedic department: the nonlocking posterior screw was directed into the spine of the scapula, and the nonlocking anterior screw was directed into the glenoid body (Fig. 1).

\subsection{Experimental setup}

When the holes of the two nonlocking screws were achieved, we removed the Aequalis baseplate and replaced it with an instrumented one, specifically developed for this study. This instrumented baseplate had the same dimensions as the Aequalis baseplate, but had only one central hole, for one nonlocking screw. This hole had the same geometry as the two nonlocking screws holes of the Aequalis baseplate. The

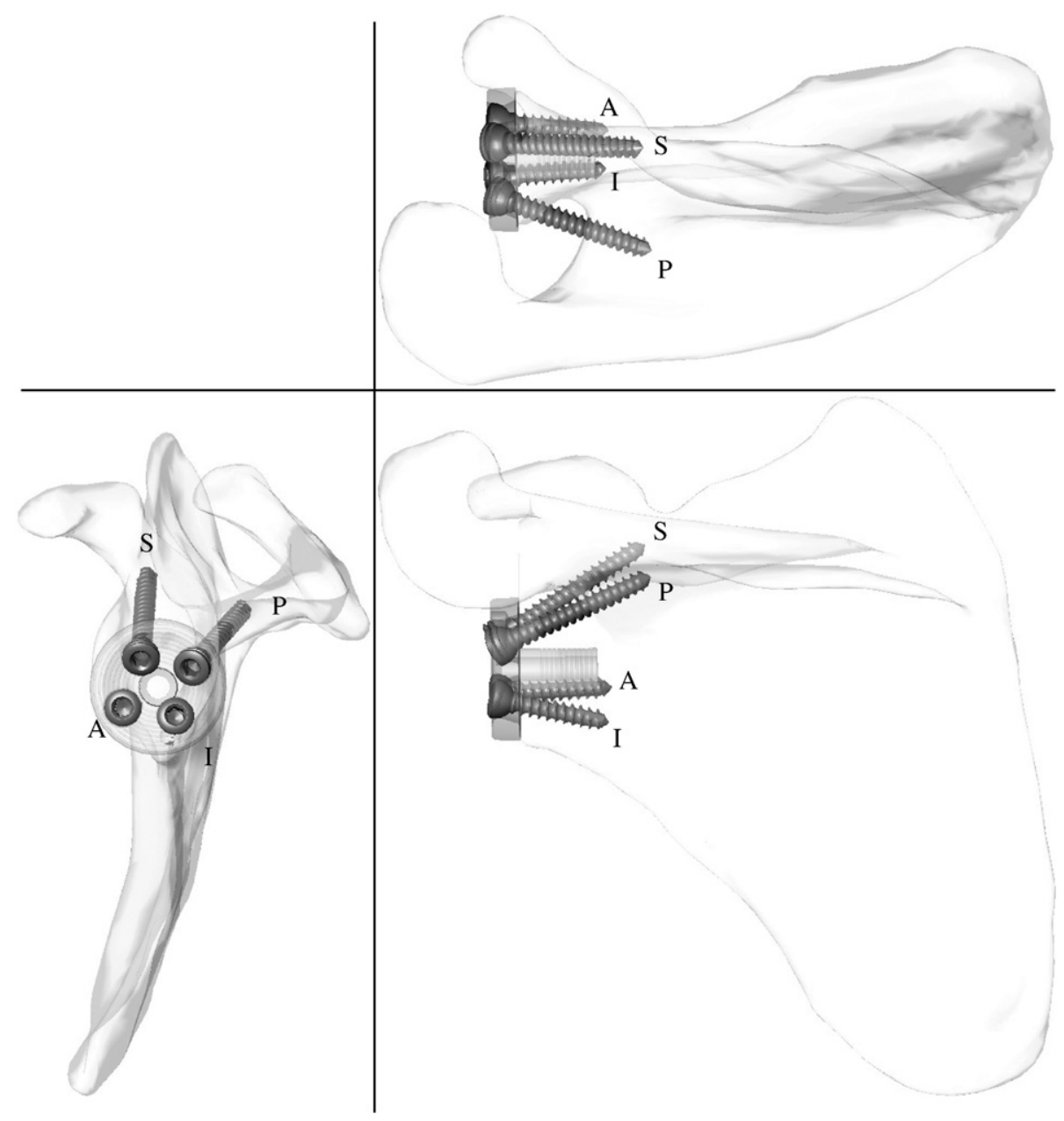

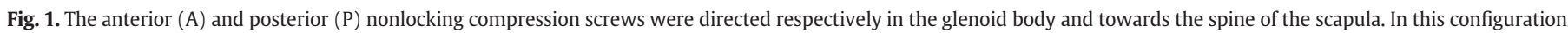
the inferior (I) and superior (S) locking screws are respectively located in the pillar and in the base of the coracoid. 
instrumented baseplate was formed of two plates enclosing three thin force captors (FlexiForce, Tekscan, MA, USA) that were positioned between the central hole and the external border of the baseplate, every $120^{\circ}$ around the central hole (Fig. 2). To provide an optimal contact between the three force captors and the two plates, the captors were placed on small cylindrical supports fixed to one plate. The tightening screw force was the sum of the forces measured by the three sensors. Rotation between the two plates was prevented by two spikes, fixed on one plate and sliding in two opposite guiding holes in the other plate. We also developed a stand to fix the scapula and to align the screw axis with the screwdriver axis. The stand was composed of a fixation device for the scapula, and an axis allowing the screwdriver to slide freely along its longitudinal axis. We linked the screwdriver to a digital torque meter (Centor W Star, Andilog, France) and a digital custom-made device to measure the screwing angle. The torque meter required a manual action. The setup allowed for a manual insertion of the screws, as the surgeons do in the operation room. The tightening force, tightening torque and screwing angle were recorded on a computer.

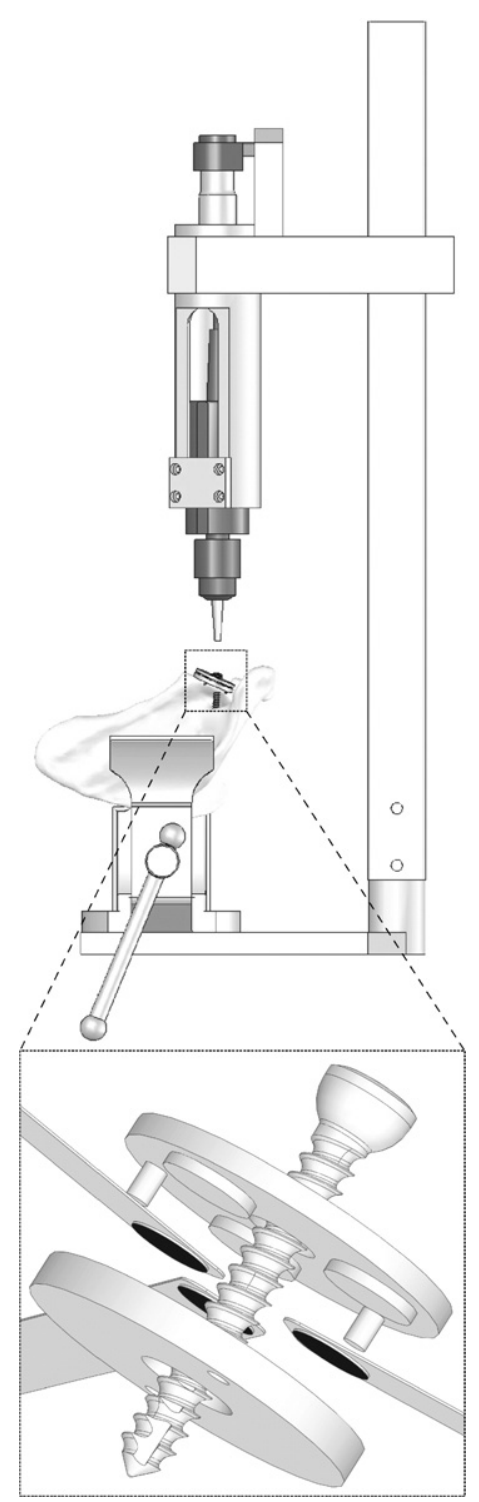

Fig. 2. Stand designed for the measurements. It supported a sliding torque meter, completer by a digital goniometer. The force was measured by an instrumented baseplate (zoom view) enclosing 3 thin FlexiForce captors (black disks). The two plates could slide along two guiding pegs to avoid relative rotation between them.

\subsection{Experimental protocol}

The same following protocol was used successively for the anterior and the posterior nonlocking screws. Each scapula was fixed in the stand such that the screw axis was aligned with the sliding screwing axis of the stand. The instrumented baseplate was placed on the glenoid surface, such that the central hole of the baseplate coincided with the pre-drilled hole of the screw. The baseplate was then slightly fixed to the glenoid bone with two self-tapping screws (diameter $2 \mathrm{~mm}$, length $10 \mathrm{~mm}$ ), that did not compress the plate but only avoided its rotation during screwing. The screw length was chosen to best fit the pre-dilled hole, in the same way as in the operation room. Before starting the measurements, the screw was screwed until abutment of its head with the baseplate. From this moment, the captors of the instrumented baseplate started to measure some force. Then, the measurement started. The surgeon continued to screw, and started to tight the screw within the glenoid bone, in the same way as in the operation room. From this moment, the tightening force $F$, tightening torque $M$ and screwing angle $\alpha$ were continuously recorded until failure of the bone. When the measurement was achieved, the screw and baseplate were removed. During the manual screwing by the surgeon, we also recorded at which angle $\alpha_{\text {surg }}$ the surgeon would stop screwing. This measurement provided the F-Alpha and M-Alpha diagrams (tightening force $F$ and tightening torque $M$ versus turn angle $\alpha$ ), marked with the surgical stop angle $\alpha_{\text {surg. }}$. The maximal tightening force $F_{\max }$ and tightening torque $M_{\max }$ were obtained from the F-Alpha and M-Alpha diagrams and corresponded to bone failure. The surgical tightening force $F_{\text {surg }}$ and tightening torque $M_{\text {surg }}$ were also obtained from the F-Alpha and $M$-Alpha diagrams and corresponded to the surgical stop angle $\alpha_{\text {surg. }}$. F-Alpha and $M$-Alpha diagrams were obtained for the 2 nonlocking screws of the 6 scapulae, providing 12 experimental curves. The first three scapulae were used to setup the measurement of the surgical stop angle $\alpha_{\text {surg. }}$. Therefore, the surgical stop angle $\alpha_{\text {surg, and thus the surgical }}$ tightening force $F_{\text {surg }}$ and tightening torque $M_{\text {surg }}$ were only obtained for the other three scapulae, providing 6 values.

\subsection{Micro-CT analysis}

After the mechanical test, we isolated the glenoid part from the entire scapula and enclosed it within a thin plastic wrap to preserve hydration. We micro-scanned the glenoid samples at a resolution of $36 \mu \mathrm{m}$, at $80 \mathrm{kV}, 120 \mathrm{~mA}$ and with a $1 \mathrm{~mm}$ Al filter (1076 $\mu \mathrm{CT}$, Skyscan, Belgium). Bone mineral density was calibrated with two phantoms $\left(0.25\right.$ and $\left.0.75 \mathrm{~g} / \mathrm{cm}^{3}\right)$ of hydroxyapatite provided by Skyscan. Images reconstruction and analysis were performed with Skyscan software (CTAn). We defined a cylindrical region of interest of $6 \mathrm{~mm}$ diameter (screw diameter + thread depth) around each screw and we measured bone volume (BV) within this region, considering a minimum threshold of $0.05 \mathrm{~g} / \mathrm{cm}^{3}$ (Fig. 3). We also measured the bone mineral density (BMD) within the bone volume (BV).

\subsection{Statistical analysis}

We tested the hypotheses that $F_{\max }, M_{\max }, F_{\text {surg }}$ and $M_{\text {surg }}$ are linearly correlated to BV. For any significant correlation, we calculated the confidence and prediction intervals about the regression line. All hypotheses were tested with a Student's $t$-test and a significance level $p<0.05$.

\section{Results}

All measurements and statistical results are given in Table 1.

\subsection{F-Alpha and M-Alpha diagrams}

We obtained the F-Alpha and M-Alpha diagrams (Fig. 4) for the 12 screws. Each screw presented the same behavior. After a first alignment 

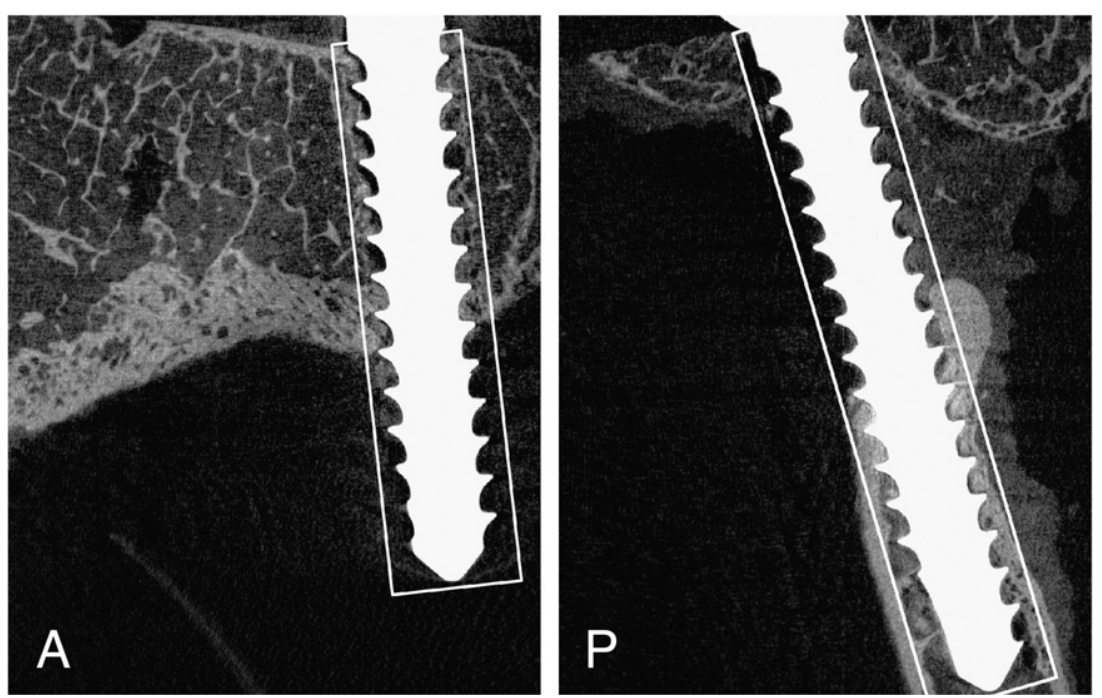

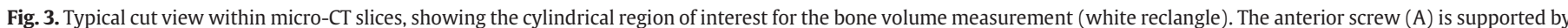

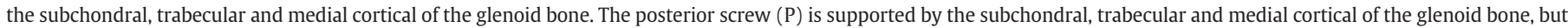
also by the cortical basis of the spine of the scapula.

phase, there was a quasi-linear tightening phase, a post-yield phase and then the failure. The alignment phase required approximately one screwing turn, and the tightening phase (until failure) another turn.

\subsection{Bone volume and density}

BV around the screws varied from 55.0 to $425.0 \mathrm{~mm}^{3}$, with an average value of 200.5 and a standard deviation of 109.2. Within BV, BMD varied from 0.27 to $0.46 \mathrm{~g} / \mathrm{cm}^{3}$, with an average of 0.37 and a standard deviation of 0.05 .

\subsection{Tightening force}

In average, $F_{\max }$ was $350 \mathrm{~N}$ and it was statistically correlated $(p=0.0008)$ with $\mathrm{BV}$. The correlation equation was $F_{\max }=159.2+$ $0.95 \mathrm{BV}$, with a correlation coefficient $R=0.83$. Confidence and

\section{Table 1}

Experimental measurement of bone volume $(\mathrm{BV})$, surgical force $\left(F_{\text {surg }}\right)$, maximal force $\left(F_{\max }\right)$, surgical torque $\left(M_{\text {surg }}\right)$ and maximal torque $\left(M_{\max }\right)$ for the 6 anterior $(1 \mathrm{~A}-6 \mathrm{~A})$ and posterior (1P-6P) screws. Since the first 6 screws were used to design the measurement protocol of the surgical stop angle $\alpha_{\text {surg }}, F_{\text {surg }}$ and $M_{\text {surg }}$ were not obtained for these screws. Besides, $M_{\max }$ for screw 1P could not be obtained because of a technical problem. This table also contains the average, standard deviation of each measured quantities. The last 4 rows are the slope, intercept, correlation coefficient, and $p$ value of the linear correlation between BV and the other quantities.

\begin{tabular}{lclllll}
\hline Screw & $\begin{array}{l}\mathrm{BV} \\
\left(\mathrm{mm}^{3}\right)\end{array}$ & $\begin{array}{l}\mathrm{BMD} \\
\left(\mathrm{g} / \mathrm{cm}^{3}\right)\end{array}$ & $\begin{array}{l}F_{\text {surg }} \\
(\mathrm{N})\end{array}$ & $\begin{array}{l}F_{\max } \\
(\mathrm{N})\end{array}$ & $\begin{array}{l}M_{\text {surg }} \\
(\mathrm{Nm})\end{array}$ & $\begin{array}{l}M_{\max } \\
(\mathrm{Nm})\end{array}$ \\
\hline 1A & 54.9 & 0.267 & - & 298.0 & - & 2.4 \\
2A & 117.7 & 0.416 & - & 225.0 & - & 1.0 \\
3A & 71.0 & 0.368 & - & 179.0 & - & 0.6 \\
1P & 201.8 & 0.350 & - & 445.0 & - & - \\
2P & 333.7 & 0.455 & - & 380.0 & - & 1.8 \\
3P & 425.0 & 0.395 & - & 545.0 & - & 3.0 \\
4A & 139.6 & 0.452 & 80.3 & 201.5 & 0.8 & 1.1 \\
5A & 167.4 & 0.325 & 137.8 & 317.3 & 0.9 & 1.5 \\
6A & 211.4 & 0.355 & 226.7 & 285.0 & 1.9 & 2.1 \\
4P & 184.0 & 0.367 & 101.6 & 320.4 & 0.5 & 1.3 \\
5P & 287.6 & 0.365 & 181.1 & 503.1 & 1.9 & 4.7 \\
6P & 268.1 & 0.360 & 388.0 & 505.0 & 2.2 & 3.2 \\
Mean & 201.5 & 0.373 & 185.9 & 350.4 & 1.3 & 2.1 \\
Std Dev & 109.22 & 0.053 & 112.35 & 124.75 & 0.72 & 1.18 \\
Slope & & & 1.164 & 0.949 & 0.008 & 0.006 \\
Intercept & & & -49.437 & 159.161 & -0.307 & 0.830 \\
$R$ & & & 0.62 & 0.83 & 0.68 & 0.59 \\
$p$ & & \multicolumn{7}{c}{0.183095} & 0.000814 & 0.134886 & 0.055126 \\
\hline
\end{tabular}

prediction limits were calculated for this correlation (Fig. 5). $F_{\text {surg }}$ was not statistically correlated to $\mathrm{BV}(p=0.1831)$. In average, $F_{\text {surg }}$ was $51 \%$ of $F_{\text {max. }}$

\subsection{Tightening torque}

$\mathrm{M}_{\max }$ was not statistically correlated to BV $(p=0.0551)$, nor was $M_{\text {surg }}(p=0.1349)$. In average, $M_{\text {surg }}$ was $60 \%$ of $M_{\text {max }}$. The (optimal) surgical tightening torque could not be determined from BV, neither from the shape of the M-Alpha curve.

\section{Discussion}

Although the initial problem of reversed shoulder prostheses has been improved by the Grammond design (Boileau et al., 2005), the determination of the optimal fixation of the glenoid baseplate remains an open question. Most reversed prostheses use peripheral fixation screws, but the ideal positioning of the screws is not known yet, nor the optimal number of screws. This problem was only recently analyzed with synthetic bone or numerical modeling, and the stabilizing effect

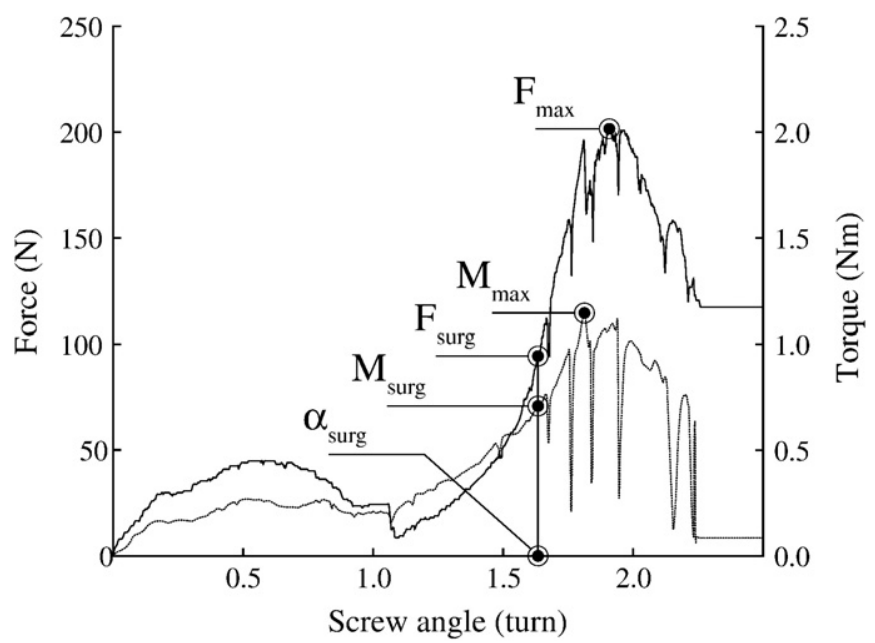

Fig. 4. Typical measurements of F-Alpha (continuous) and $M$-Alpha (dotted) diagrams,

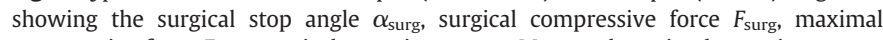
compressive force $F_{\max }$, surgical screwing torque $M_{\text {surg }}$ and maximal screwing torque $M_{\max }$ nonlocking compression screws. 


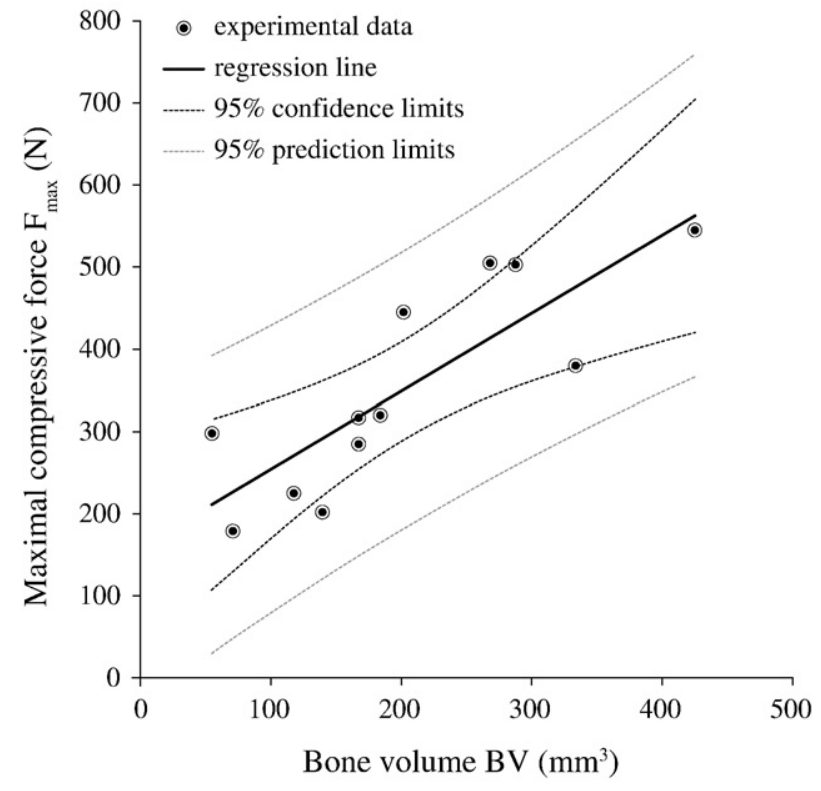

Fig. 5. Maximal compressive force vs. bone volume surrounding the nonlocking compression screws, with the regression line, 95\% prediction limits and 95\% confidence limits.

caused by the tightening force of nonlocking screws was never documented or considered. Our measurements on cadaveric scapulae provided a correlation between the maximal tightening screw force and the surrounding bone volume.

The experimental setup developed for this study provided the simultaneous measurement of the tightening force, tightening torque, and screwing angle of the two nonlocking compressive screws of the Aequalis reversed prosthesis. Since the screw holes were pre-drilled and measurement was started from the abutment of the screw head with the instrumented baseplate, we actually measured the tightening force and the tightening torque, and not the insertion force and torque, which are more commonly measured. There was in fact no force applied on the screwdriver during this measurement, but only a torque. Force and torque measurements were related to bone volume within a cylindrical region of interest surrounding the screw. Because of the complex glenoid anatomy, bone volume was indeed a reliable estimate of the bone support, instead of the screw penetration or (average) bone mass density (BMD). A classical measure of the bone support with a clinical CT would indeed not be relevant in this situation since the bone volume is highly non homogeneous around the screws. All screws were indeed surrounded partly by trabecular bone, partly by cortical bone, or partly outside the scapular bone. We also measured the BMD within the BV, to assess its consistency with other published measurements (Lehtinen et al., 2004). Because of the variation of bone support (cortical, trabecular, and no bone), the limited volume of the region of interest, and the limited number of bone samples, we did not test other local bone architecture parameters such as trabecular thickness, porosity, or anisotropy. The diameter of the cylindrical region of interest was chosen such that it contains the screws plus a thread depth. We indeed assumed that the bone quality within that region affects the screw fixation.

The surgical tightening force was not statistically correlated to bone volume, but it was in average approximately $50 \%$ of the maximal tightening force, which was correlated to the bone volume. This result is quite natural according to the F-Alpha diagram, and can be seen as a safety factor. The maximal tightening force could however be used as a superior limit prediction, with the half value being an estimate of the surgical tightening force.

Neither maximal nor surgical tightening torque was statistically correlated to bone volume, although maximal torque was close to the significance criteria $(p=0.055)$. Surgical torque could thus not be determined from bone volume, but neither from the M-Alpha curve as it is done in bolt mechanics. This second option would require a smoother and more accurate $M$-Alpha diagram to estimate the yield torque. Our $M$ Alpha diagrams were indeed altered by torque and force drops, which corresponded to pauses during the manual screwing process. These experimental issues did not alter the measurement of maximal and surgical tightening force and torque. This prediction might however be feasible with a more precise experimental setup. The M-Alpha diagram showed indeed a typical behavior, starting with an alignment phase, a quasi-linear tightening phase, a yield point, and the failure. The surgeon did not stop screwing at the same level of torque, but surprisingly stopped rather consistently (but not statistically significantly) at $50 \%$ of the failure torque. We think that the surgeon cannot predict the upcoming failure, but may rather feel a decrease in screwing resistance (delta torque/delta alpha), which we can observe (but not quantify) in the $M$-alpha diagram (Fig. 4). We also think that the limited number of measurements and the measurement variability mainly explain the difficulty to obtain a significant correlation between the surgical force and the bone volume. In mechanical engineering the tightening torque is often recommended to be $50 \%$ of the stripping (failure) torque. It was here measured at $60 \%$.

Various measurements of force, torque and bone quality have been reported in many studies. However, the quantities measured are not the same as the present work, which makes the comparison not easy. For example, pullout force is not equivalent to tightening force, or screwing torque to tightening torque, or bone mass density to bone volume. The relationship between pullout force, peak initial insertion torque and BMD was examined on 30 cervical spines (Ryken et al., 1995). A comparison of unicortical and bicortical screws provided similar BMD, but higher force and torque for bicortical screws. There was a linear correlation between peak torque, pullout force and BMD. The average pullout force, peak torque and (areal) BMD were respectively $210 \mathrm{~N}$, $0.367 \mathrm{Nm}$ and $0.79 \mathrm{~g} / \mathrm{cm}^{2}$. Our maximal tightening force was not very different from this peak pullout force, but our tightening torque could not be compared, since it was differently defined. With a simple transformation of the measured volumetric bone density into a real BMD (Sran et al., 2005), our average BMD was $0.95 \mathrm{~g} / \mathrm{cm}^{2}$, which is also comparable to this spine study. In another study, the range of tightening force and tightening torque of compression screws measured in scaphoid cadaver bone was in the same range as our measurements (Wheeler and McLoughlin, 1998). Other measurements of tightening torque and associated BMD of bicortical screws on cadaveric femora were still in the same range as our values (Kincaid et al., 2007). Another cadaveric measurement of tightening force and torque reported that maximal force and torque occurred at the same time during the screwing, as we observed in the F-Alpha and M-Alpha diagrams (Perren, 1976). Intraoperative measurements of insertional torque of pedicle screw associated to BMD compared also to our measurements (Okuyama et al., 2000). Using synthetic foam bone, tightening force and torque were measured on various bone screws and compared to theoretical predictions, providing a relation between tightening and pullout force (Brown et al., 2000). Their values were again in the same range as ours.

The present results are the first measurements of tightening screw force of reversed shoulder prostheses on human cadaver bone. Since nonlocking screws of reversed prostheses mainly provide initial stability though compression, it was important to measure tightening force instead of pullout force. F-Alpha and $M$-Alpha diagrams were very useful to understand the link between the maximal and surgical value of the tightening force and torque during the screwing process and might eventually help in optimizing the screwing process. Although statistical correlation between all variables could not be obtained, the relationship between maximum force and bone volume will be useful to further optimize baseplate stability.

The main limitation of the present study is certainly the number of measurements. With 6 scapulae, we obtained 12 F-Alpha and M-Alpha diagrams and 12 measures of the associated bone volume around the screws. However, since the three first scapulae were used to setup the 
protocol of the surgical force and torque measurement, only 6 measures were obtained. Measurement might also have some uncertainty compared to the values on real patients since the screwing device and surgeon position were not exactly the same as in the operating room, although the surgical technique and instrumentation was the same as the one used in the operation room. In addition, measurements were limited to the Aequalis prosthesis, but nonlocking screws of other prostheses are rather similar. The scapulae used in this study were fixed with a formalin solution. Several studies however reported that $10 \%$ formalin fixation have no statistical effect, or a limited effect (10\%), on bone strength (Cowin, 2001). The stress relaxation of bone might also decrease the estimated tightening force by 10 to 20\% (Goto et al., 1999; Inceoglu et al., 2004). At another time scale, bone remodeling process will also reduce this force to nearly zero. The proper fixation of the baseplate is however mainly important for the early (days) biological integration at the bone-metal interface. Regarding all above limitations, we may assume that the correlation between maximal force and bone volume, and the ratio between surgical and maximal force were still a good estimation of the clinical situation.

\section{Conclusions}

To conclude, the obtained relationship between tightening force and bone volume should be helpful to further improve the optimal number and position of the nonlocking screws that stabilize the baseplate of reversed shoulder prostheses. It could be indeed used to predict tightening force of another screw positioning from micro-CT images of cadaveric scapulae, to actually account for the tightening force in experimental tests, or for more complex stability predictions of numerical models.

Finally, these cadaveric or numerical results could then be extrapolated for clinical use, providing general recommendations on the screw positioning, which is still a debate.

\section{Acknowledgments}

We thank Tornier (Tornier Inc., Edina, MN) for its financial and technical support. The author also thank Prof Jean-Pierre Hornung (Anatomy Department, University Hospital of Lausanne), who provided the cadaveric scapulae.

\section{References}

Bohsali, K.I., Wirth, M.A., Rockwood Jr., C.A., 2006. Complications of total shoulder arthroplasty. J. Bone Joint Surg. Am. 88 (10), 2279-2292.

Boileau, P., Watkinson, D., Hatzidakis, A.M., Hovorka, I., 2006. Neer Award 2005: the Grammont reverse shoulder prosthesis: results in cuff tear arthritis, fracture sequelae, and revision arthroplasty. J. Shoulder Elbow Surg. 15 (5), 527-540.

Boileau, P., Watkinson, D.J., Hatzidakis, A.M., Balg, F., 2005. Grammont reverse prosthesis: design, rationale, and biomechanics. J. Shoulder Elbow Surg. 14 (1 Suppl S), 147S-161S.
Brown, G.A., McCarthy, T., Bourgeault, C.A., Callahan, D.J., 2000. Mechanical performance of standard and cannulated $4.0-\mathrm{mm}$ cancellous bone screws. J. Orthop. Res. 18 (2), 307-312.

Chebli, C., Huber, P., Watling, J., Bertelsen, A., Bicknell, R.T., Matsen 3rd, F., 2008. Factors affecting fixation of the glenoid component of a reverse total shoulder prothesis. J Shoulder Elbow Surg. 17 (2), 323-327.

Codsi, M.J., Iannotti, J.P., 2008. The effect of screw position on the initial fixation of a reverse total shoulder prosthesis in a glenoid with a cavitary bone defect. J. Shoulder Elbow Surg.

Cowin, S.C., 2001. Bone Mechanics Handbook. CRC Press, Boca Raton, FL.

Goto, T., Sasaki, N., Hikichi, K., 1999. Early stage-stress relaxation in compact bone. J. Biomech. 32 (1), 93-97.

Guery, J., Favard, L., Sirveaux, F., Oudet, D., Mole, D., Walch, G., 2006. Reverse total shoulder arthroplasty. Survivorship analysis of eighty replacements followed for five to ten years. J. Bone Joint Surg. Am. 88 (8), 1742-1747.

Harman, M., Frankle, M., Vasey, M., Banks, S., 2005. Initial glenoid component fixation in "reverse" total shoulder arthroplasty: a biomechanical evaluation. J. Shoulder Elbow Surg. 14 (1 Suppl S), 162S-167S.

Hopkins, A.R., Hansen, U.N., Bull, A.M., Emery, R., Amis, A.A., 2008. Fixation of the reversed shoulder prosthesis. J. Shoulder Elbow Surg. 17 (6), 974-980.

Humphrey, C.S., Kelly 2nd, J.D., Norris, T.R., 2008. Optimizing glenosphere position and fixation in reverse shoulder arthroplasty, Part Two: The three-column concept. J. Shoulder Elbow Surg. 17 (4), 595-601.

Inceoglu, S., McLain, R.F., Cayli, S., Kilincer, C., Ferrara, L., 2004. Stress relaxation of bone significantly affects the pull-out behavior of pedicle screws. J. Orthop. Res. 22 (6), 1243-1247.

Kienapfel, H., Sprey, C., Wilke, A., Griss, P., 1999. Implant fixation by bone ingrowth. J. Arthroplasty 14 (3), 355-368.

Kincaid, B., Schroder, L., Mason, J., 2007. Measurement of orthopedic cortical bone screw insertion performance in cadaver bone and model materials. Exp. Mech. 47 (5), 595-607.

Kwong, L.M., O'Connor, D.O., Sedlacek, R.C., Krushell, R.J., Maloney, W.J., Harris, W.H., 1994. A quantitative in vitro assessment of fit and screw fixation on the stability of a cementless hemispherical acetabular component. J. Arthroplasty 9 (2), 163-170.

Lehtinen, J.T., Tingart, M.J., Apreleva, M., Warner, J.J., 2004. Total, trabecular, and cortical bone mineral density in different regions of the glenoid. J. Shoulder Elbow Surg. 13 (3), 344-348.

Okuyama, K., Abe, E., Suzuki, T., Tamura, Y., Chiba, M., Sato, K., 2000. Can insertional torque predict screw loosening and related failures? An in vivo study of pedicle screw fixation augmenting posterior lumbar interbody fusion. Spine 25 (7), 858-864.

Perren, S.M., 1976. Force measurements in screw fixation. J. Biomech. 9 (11), 669-675

Roche, C., Flurin, P.H., Wright, T., Crosby, L., Hutchinson, D., Zuckerman, J., 2008. Effect of varying screw configuration and bone density on reverse shoulder glenoid fixation following cyclic loading. 54th Annual Meeting of the Orthopaedic Research Society. San Francisco.

Ryken, T.C., Clausen, J.D., Traynelis, V.C., Goel, V.K., 1995. Biomechanical analysis of bone mineral density, insertion technique, screw torque, and holding strength of anterior cervical plate screws. J. Neurosurg. 83 (2), 325-329.

Sirveaux, F., Favard, L., Oudet, D., Huquet, D., Walch, G., Mole, D., 2004. Grammont inverted total shoulder arthroplasty in the treatment of glenohumeral osteoarthritis with massive rupture of the cuff. Results of a multicentre study of 80 shoulders. J. Bone Joint Surg. Br. 86 (3), 388-395.

Soballe, K., Hansen, E.S., B-Rasmussen, H., Jorgensen, P.H., Bunger, C., 1992. Tissue ingrowth into titanium and hydroxyapatite-coated implants during stable and unstable mechanical conditions. J. Orthop. Res. 10 (2), 285-299.

Sran, M.M., Khan, K.M., Keiver, K., Chew, J.B., McKay, H.A., Oxland, T.R., 2005. Accuracy of DXA scanning of the thoracic spine: cadaveric studies comparing BMC, areal BMD and geometric estimates of volumetric BMD against ash weight and CT measures of bone volume. Eur. Spine J. 14 (10), 971-976.

Wheeler, D.L., McLoughlin, S.W., 1998. Biomechanical assessment of compression screws. Clin. Orthop. Relat. Res. (350), 237-245.

Wierks, C., Skolasky, R.L., Ji, J.H., McFarland, E.G., 2009. Reverse total shoulder replacement: intraoperative and early postoperative complications. Clin. Orthop. Relat. Res. 467 (1) 225-234. 